\title{
Perceptions, beliefs, and needs of Japanese people with knee osteoarthritis during conservative care: A qualitative study
}

Daisuke Uritani ( $\nabla$ d.uritani@kio.ac.jp )

Kio University

\section{Akane Ikeda}

Kio University

Toru Shironoki

Kio University

Kentaro Matsubata

Kio University

\section{Yuto Mutsura}

Kio University

\section{Tadashi Fujii}

Kashiba Asahigaoka Hospital

\section{Koji Ikeda}

Naragakuen University

\section{Research Article}

Keywords: Osteoarthritis, Knee, Qualitative study, Patient Care Management

Posted Date: May 27th, 2021

DOl: https://doi.org/10.21203/rs.3.rs-558422/v1

License: (9) (i) This work is licensed under a Creative Commons Attribution 4.0 International License. Read Full License 


\section{Abstract}

Background Patients' perceptions and beliefs of disease could be influenced by their lifestyle and culture. Although it is important to understand their perceptions and beliefs toward disease to prevent and manage osteoarthritis $(\mathrm{OA})$ through conservative care, this topic has not been investigated in Japanese people with knee OA. Therefore, this qualitative study aims to clarify how Japanese patients with knee OA experience and perceive their symptoms and disabilities, and how they face them during conservative care.

Methods Participants were recruited by purposive sampling. Face-to-face, semi-structured interviews were conducted with nine patients ( 2 men and 7 women, mean age, $74.3 \pm 5.5$ years) with knee OA until data saturation was reached. Interview data comprised participants' accounts of particular personal experiences of living with knee OA, including their perceptions and attitudes toward knee OA-related symptoms and disabilities. Two physiotherapists (one with extensive experience conducting qualitative studies) and four physiotherapy students conducted the interviews. Recorded interview data were transcribed verbatim in Japanese. Data analysis, including developing a coding scheme, was conducted based on a grounded theory approach.

Results Six categories were extracted from the data: "self-analysis on the cause of knee OA," "difficulties in daily life due to knee symptoms," "psychological barrier," "how to deal with knee pain and difficulty in moving," "importance of connecting with others," and "information considered useful to cope with knee OA." Japanese patients with knee OA desired evidence-based information and to connect with other people in the same situation to solve problems related to their condition.

Conclusions To address patients' concerns, medical professionals should carry out careful interviews and obtain information regarding patients' past experiences, and understand their experiences related to knee $O A$. Symptoms and difficulties experienced by patients with knee OA should be managed by evidencebased information integrating their perceptions and beliefs toward knee OA.

\section{Background}

Knee osteoarthritis (OA) is a prevalent, costly chronic condition characterized by physical symptoms and functional limitation in older people [1-3]. In Japan, more than $60 \%$ of adults aged 60 years or above suffer from radiographic knee $O A$, and more than $26 \%$ adults aged 60 years or above have symptomatic knee OA [4]. Self-management, OA education, and regular exercise during conservative care are important for patients with knee OA to improve their condition $[5,6]$. Medical professionals should keep this in mind when proposing comprehensive management of pain/symptoms experienced by knee OA patients through various means, from therapeutic exercises to patient education, during conservative care.

Using the tools available to them, healthcare professionals must understand how patients perceive knee $\mathrm{OA}$ and the feasibility of self-management of symptoms, and understand patients' information-related needs about knee OA [7]. Previous studies have shown that a therapist's inability to understand how 
patients perceive their own situation is a key barrier to successful public health activities $[8,9]$. Therefore, understanding how patients perceive their own symptoms is an important first step in developing interventions aimed at preventing and managing $O A$ at conservative care.

Patients' perceptions and attitudes toward the disease reflect their experiences. People aged 35-65 years suffering from painful knees believed that OA's progression could be prevented or delayed, and did not consider OA as a natural or inevitable event [10]. Meanwhile, people aged 60 years and above perceive OA as a naturally occurring part of aging [11-13]. These findings suggest that patients with different backgrounds have different perceptions toward knee OA.

Previous studies indicated that people's attitude toward dealing with pain [14], health beliefs, and selfcare [15] were varied owing to differences in lifestyle and culture between Japan and other countries. Therefore, perceptions toward knee OA may also differ between Japan and other countries. For successful conservative management of patients with knee $O A$, healthcare professionals must understand patients' perceptions and beliefs toward knee OA and their needs. While patients' perceptions and attitudes toward knee OA have been reported and synthesized in Western and other Asian countries [16-18], it has not been investigated in Japan. Therefore, the purpose of this qualitative study was to explore Japanese patients' perceptions and beliefs towards knee OA and their needs during conservative care. A qualitative research was adopted because this study was not limited to simply summarizing the data obtained from the interviews. The aim was to elucidate the relationship between the phenomena illustrated by the data and to generate a theory depicting the processes through which the phenomena occur [19].

\section{Methods}

This study employed a cross-sectional, qualitative design. It adhered to the standards for reporting qualitative research [20].

\section{Participants}

Study participants comprised patients with knee OA from different regions in Japan. Purposive sampling according to the inclusion criteria determined by the research purpose was carried out to obtain rich, relevant, and diverse data pertinent to the research question [21]. Inclusion criteria were a confirmed diagnosis of knee OA in one or both knees, aged over 50 years, and self-reported experience of pain or disability. We interviewed patients who seemed extroverted to gather detailed and more descriptive information. Patients suspected of having cognitive decline or communication difficulties, although not specifically tested, were excluded. Recruitment of study participants was conducted by an orthopaedic surgeon or physical therapists at facilities cooperated for this research.

\section{Interviews and data collection}


The interviewers were two physiotherapists and four physiotherapy students. All interviewers were Japanese (one woman and five men). The two physiotherapists had 19 (DU) and 26 (KI) years of work experience in physiotherapy, including musculoskeletal physiotherapy. Physiotherapist KI was also an experienced qualitative researcher. The physiotherapy students ( $\mathrm{Al}, \mathrm{TS}, \mathrm{KM}, \mathrm{YM}$ ) were in the final year of their baccalaureate degree program and had completed clinical clerkship in their undergraduate curriculum. Interviews were held in an isolated room at the orthopaedic clinic where we recruited participants and at a meeting room at the university to which DU belongs. Data were collected from February to August 2019. One interviewer (DU) conducted all interviews to ensure consistency with or without other researchers. The four physiotherapy students were trained by researcher $\mathrm{KI}$ in advance. Participants were unknown to the interviewers prior to recruitment. Each interview lasted approximately 1-1.5 hours. Participants were interviewed one at a time, except two participants who were a couple, and in a face-to-face manner. Interview data comprised participants' particular personal experiences of living with knee OA to clarify the perceptions and beliefs toward symptoms and disabilities about knee OA, and their needs to deal with their symptoms and difficulties during conservative care. Semi-structured interviews were conducted using an interview guide created based on previous studies [10, 22-25] (Table 1) and comprising open-ended questions to elicit participants' perspectives of their experiences and ideas regarding knee $\mathrm{OA}$. Guided questions encouraged participants to describe their experiences and thoughts regarding knee OA during conservative care. The interviews were kept flexible to allow participants to talk about what they deemed important. Additionally, interesting or important statements from participants previously interviewed were reflected as questions to subsequent participants. All the interviews were recorded using an IC recorder (ICD-SX950, SONY, Tokyo, Japan) and recorded data were managed using a software program (Sound Organizer Ver.1.6). Observational memos, including contextual characteristics, atmosphere, and relevant non-verbal expressions, were also recorded. No repeat interviews were conducted with the same participants.

Recruitment, data collection, and data analysis occurred concurrently to enable data to inform subsequent interviews and to cease recruitment once theoretical saturation was achieved. The criterion for saturation was that no new theme was identified in the new interview after consensus among the researchers. Recorded data were transcribed verbatim in Japanese after the interview by five interviewers (DU, Al, TS, KM, YM). Recorded data from each interview were divided into several parts. Annotations were noted to indicate vocal inflections and utterances by the interviewer where necessary. The transcripts were crosschecked against the recorded data for completeness and accuracy by another interviewer.

\section{Analysis}

Data analysis was conducted based on a grounded theory approach [26, 27]. Transcripts were divided into several sentences according to the content and context of the statements, and coded individually. These coded parts were listed and clustered into groups to form initial small categories (subcategories). This was done to create draft categories for the data obtained from the interview of the first participant. After the interview of the second participant, divided parts of transcripts were coded and already-formed 
draft categories were applied. These procedures formed an iterative process that involved integrating closely related codes, separating other more complex ones into separate elements, and developing new categories where necessary. Relationships between and within small categories emerging from this process were explored with increasingly higher levels of conceptualization. Small categories across all transcripts were then listed and clustered into larger categories based on similarity and overlap. This grouping was refined to identify main themes. Coding and categorizing were crosschecked by all researchers with all disagreements resolved through regular discussions.

\section{Results}

Interview data were obtained from nine participants with knee OA (mean age $=74.3 \pm 5.5$ years, 2 men, 7 women) (Table 2). Six participants were recruited from among outpatients at a general hospital, of which, two participants were from an orthopaedic clinic, and one participant from among attendees of a community-based health care program. Three participants had undergone total knee arthroplasty (TKA) in the past. Participants who had undergone TKA were also interviewed about their experiences of conservative treatment before TKA.

Six categories were developed from the data. The categories are presented below followed by the subcategories. They are described with illustrative quotes presented throughout the text using pseudonyms that match Table 2. Detailed illustrative quotes are given in the Additional file. Figure 1 depicts the relationship between categories.

\section{Self-analysis on the cause of knee $O A$}

\section{Overwork/overload}

Participants believed that overworking their knees in youth was responsible for their current painful and disabled knee condition. They suspected that the effects of other body parts, especially the condition of feet and ankles, or personal characteristics of walking and movement might be associated with their overloaded/overworked knee joint.

"I think I've overworked my knees, when I thought about various causes of knee OA ...I think I was walking more than others. I was guiding customers at work. That's the reason of knee OA that comes to mind." (A) "I think that hallux valgus was progressing. So, I also think that the hallux valgus might have affected the knee." (G)

\section{Aging}

Some participants believed that aging was one of the causes of knee OA, but others did not.

"I think there is an age effect (on knee OA). When I was diagnosed with knee osteoarthritis, I thought I was finally old." (G) 
"I think many people believe that the cause of knee $O A$ is aging, but I don't think age is related to knee $O A . "(E)$

\section{Exposure to cold}

A few participants suspected that exposure to cold might have contributed to the deterioration of the condition of their knees. They cited their own constitution, overcooling their knees due to air conditioning, and their inability to cope with changes in temperature.

"I have a constitution that causes pain in my joints when my body gets cold." $(\mathrm{E})$

\section{Difficulties in daily life due to knee symptoms}

\section{Movement with bending the knee}

Participants described having difficulty in movements that required bending the knee. Especially in the Japanese lifestyle, deeply bending the knee like squatting or sitting with legs folded is required at various occasions, and there were some cases where movement was hindered.

"It's very difficult to pick up objects on the floor with bending knees." (I)

"I can no longer sit down on my heels on tatami mat." (A)

\section{Start of movement}

Difficulty in initiating movement due to knee pain or stiffness are common symptoms in patients with knee OA. The participants in this study had similar complaints.

"When I stood for a long time and started moving, I found it difficult to move." $(H)$

\section{Psychological barrier}

\section{Prudence about movement and activities}

Participants were gradually becoming more cautious about their movements to prevent increasing pain and falls. More importantly, it made them cautious about going out and interacting with others.

"I think that the experience of being unable to move my legs or having a pain in my knees is a barrier not only to my behaviour but also to my mentality. That has led to restrictions on activities." $(H)$

\section{Not wanting to bother others}

Participants reported feeling sorry for the inconvenience caused to others by leaving their jobs or roles in the community. Additionally, they thought that pain and distress should not be complained about to others and should just be tolerated. 
"I had to take a break from part-time work and teaching Tai Chi. It was the hardest thing to bother people around me." (I)

"When I complain about my pain or trouble to others, I regret saying something people don't like to hear." (G)

\section{Desire to avoid surgery}

Participants expressed their desire to avoid surgery. Those who continue treatment conservatively have anxiety/fear and negative thoughts about surgery. The idea of patience as a virtue was also reflected in the idea of surgery.

"I'm wondering if I should undergo arthroplasty, but I'm trying to delay it as much as possible. If possible, I would like to avoid arthroplasty ..." (A)

"It may be due to the characteristics of Japanese beliefs, but it cannot be denied that undergoing surgery is regarded as a defeat to myself." (F)

\section{How to deal with knee pain and difficulty in moving}

\section{Controlling activities based on subjective sensations}

Participants relied on their own physical sensations to control their amount of exercise and physical activity. Some participants made intuitive judgments. Others used pain as an indicator of the amount of exercise and activity.

"When I go walking, I stop walking when I feel like I can't stand the pain." (D)

\section{Continuing exercise and physical activity}

Participants recognized the importance of exercise and physical activity. They participated in volunteer activities and actively engaged in personal activities such as garden maintenance. Others walked or did strength training. While recognizing the importance of activities, the difficulty of carrying them out was also acknowledged.

"I will try to walk as much as possible so that my symptoms do not get worse. I'm thinking of doing activities as long as I can move." (I)

"I affirm in my head that I have to exercise, but, in fact, it is difficult to put it into practice while reflecting on it." (F)

\section{Ingenuity to reduce knee pain and difficulty in movement}

Participants described their ingenuity in the way of daily movements, especially in walking, to reduce knee symptoms. There were also other ideas regarding equipment use, such as handrails and chairs. 
"I think how to move and walk is important to alleviate the symptoms of the knee. So, I'm trying to deal with the knee symptoms by thinking about countermeasures myself." (G)

\section{Way of thinking}

Participants considered independence and positive attitude toward life and activities important to face knee OA. They expressed that negative thoughts put them in a negative state, and positive thoughts in a positive state.

"I think that personality is also very important when dealing with knee OA. Some people are worried in advance, but I feel like it's going to be really bad in that situation." (F)

\section{Information considered useful to cope with knee $O A$}

\section{Evidence-based information}

What participants wanted most was evidence-based information. They also wanted medical professionals to appropriately guide activities and ways of thinking based on their specialized knowledge.

"I can trust evidence-based explanation and be convinced by it." (C)

\section{Informal information}

Participants gathered informal information about knee OA by word of mouth and media, such as magazines, TV, or internet. Some actively collected information from these sources.

"I often had the opportunity to learn about the doctor's reputation and the hospital's reputation in conversations with my neighbours." $(H)$

"I searched for information about knee OA from TV, magazines, and books." (E)

\section{Importance of connecting with others}

\section{Interacting with people in the same situation or in the same generation}

Participants wanted a place where they could express their painful feelings and experiences related to knee OA. They believed such opportunities would help them psychologically. Simultaneously, they emphasized the importance of a local community of people in the same situation (facing knee OA) or people of the same generation because they did not want to bother others and thought that others, including their family, could not understand their feelings unless they are parties concerned as patients with knee OA.

"I think it is important to have a place where we can vent our feelings and experiences, regardless of whether our issues can be solved. It makes me feel better, encourages me and makes me want to do my 


\section{Discussion}

To our knowledge, this is the first study to explore how Japanese patients with knee OA experience and perceive knee OA during conservative treatment. In addition, this study also clarified what they were looking for to solve their problems. Participants self-analysed the cause of knee OA (Category 1), and experienced various difficulties about movement and activity in daily life (Category 2). Thereafter, they became cautious and restrained toward certain activities, and developed a desire to not want to bother others (Category 3). Through such experiences, they found their own ways of dealing with the symptoms and difficulties of knee OA (Category 4). Additionally, they expressed a desire for evidence-based information (Category 5) and connections with others to eliminate both the physical and mental difficulties associated with knee OA (Category 6) (Figure).

We found similarities in the perceptions of Japanese participants in our study and participants from other countries [18]. The cause of knee OA such as "overwork/overload" and "aging," difficulties in daily life due to knee symptoms, psychological barriers, and the importance of connecting with others were also demonstrated in a previous systematic review [18]. Therefore, some scientific evidence in other countries could be applied to manage pain/symptoms experienced by Japanese patients with knee OA.

Meanwhile, there are some characteristics specific to Japan, such as Japanese-style movements, which were related to the participants' experiences and ideas. When self-assessing knee dysfunction, Japanese people often consider whether or not they can sit on their heels in a Japanese-style room. In Japan, some movements are very culture-specific; for example, sitting on a tatami mat. Making such movements involve self-analysis about the cause of knee $\mathrm{OA}$ and the difficulties experienced in daily life. Older Japanese people often encounter such situations during group activities and ceremonial occasions. They often avoid these situations because they do not want to bother others by not being able to perform Japanese-style movements. There are similar reports about culture-related activities in other countries $[16,22,28]$, suggesting the need to provide information for patients with knee OA to reduce their difficulty of movement in Japanese-style activities.

A few participants thought that "exposure to cold" was the cause of knee OA. In Japan, there is a belief that exposure to cold is the cause of all illnesses; a similar explanation was also found in another study [28]. Information on the effects of "coldness," and how to deal with it, must be delivered to meet the needs of Japanese patients with knee OA.

Knee OA makes patients more cautious about their activities, making them conscious of their movements and speed of movement, and in some cases avoiding movement [29]. The fear avoidance model [30] states that patient anxiety leads to inactivity, which creates a vicious cycle that exacerbates symptoms and further increases anxiety. This was commonly reported by many participants in the current study. Therefore, it is important to alleviate the difficulty of movements and activities that cause anxiety in patients from an early stage and to provide appropriate information for self-management. It can also be 
inferred that providing patients with necessary and sufficient evidence-based information will reduce anxiety and increase activity.

In addition, the patient complained of anxiety about surgery and a desire to avoid it. Previous studies have also reported that patients with knee OA were anxious about joint replacement because of concerns about the effectiveness of surgery, the risk of surgery, and the duration of recovery [12,31-33]. Providing evidence-based information about surgery as one of the future treatment options may also be necessary to reduce patients' anxiety.

From the various experiences related to knee OA as described above, participants in this study had their own beliefs and coping strategies for knee OA. Participants recognized the importance of exercise and physical activity to manage symptoms of knee OA, even though they believed that knee overuse in the past caused the current condition of knee OA. This is a different perspective since previous studies described the belief that exercise and physical activity could increase knee pain and structural damage $[28,34,35]$. Meanwhile, participants did not have a specific criterion for judging the appropriate amount of exercise and physical activity, and used their own physical sensations as the criteria. Similar reports have been found in previous studies [31, 36-39], but such self-judgment is often inaccurate [32]. There is evidence on exercise and physical activity being recommended for patients with knee $O A[5,18,36,40$, 41]. Delivering evidence-based information accurately is necessary. Based on the categories, information on activities of daily living that require knee flexion, starting movement, and appropriate amount of activity are interpreted as patient needs. By understanding patients' problems while paying attention to these issues, and applying them to the practice of guidance, more effective management of knee OA during conservative care could be expected.

Participants also recognized the importance of having a positive mindset to manage knee OA. This indicates that if we can suppress negative feelings and develop a positive attitude through appropriate guidance and information provision, it will lead to effective management of knee $\mathrm{OA}$ in conservative care.

An issue highlighted throughout the data was the importance of offering patients specific, evidencebased information. Meanwhile, previous studies in other countries demonstrated that informal rather than evidence-based information was recognized as an important resource throughout conservative care [16, $17,28,34]$. Category 4 suggests that participants wanted reliable, evidence-based information. Nonetheless, as with many previous studies [12, 17, 24, 25, 42-44], the primary information sources were informal. Participants in this study did not have sufficient opportunities to access reliable information sources and evidence-based information.

Patients' distrust of medical professionals may also be why they attach importance to informal information over evidence-based information [36]. A poor relationship between the medical professional and the patient at the beginning of treatment is detrimental for treatment, and mutual trust is important to foster a positive attitude toward conservative care [45]. Medical professionals should attend to patients' complaints from the beginning to build a relationship of trust with them. 
Interaction with others in the local community may also be effective in reducing the physical and psychological burden on patients with knee OA. Knee OA affects individuals socially, because of limited activity and participation $[18,36]$. Participants in this study wanted to connect with people who are in the same generation with the same experiences because participants believed only such people could understand their difficulties and the feeling of not wanting to bother others. Establishing a community where individuals can interact is an opportunity for patients to express their feelings and would facilitate eliminating the psychological barrier. This may be effective in finding a solution for the psychological barrier caused by knee OA. Several Japanese studies report that group exercise is easier to adhere to and more effective than individual exercise $[46,47]$. Easy participation, forming connections, and establishing a community where reliable information can be obtained, may also be important for the physical support of Japanese patients with knee OA patients.

This study has several limitations. First, the issue of generalizability. We selected sociable and eloquent participants and asked for research cooperation to increase the possibility of obtaining ample data. Some patients refused to cooperate during recruitment, and thus the data obtained were related only to the patients who participated. The results of this study are thought to reflect the experiences and perceptions of the older generation, but different perceptions and experiences may be found in younger patients, as shown in previous studies [10]. The slight gender differences in our sample (more women) might have also affected the generalizability of the results and the representativeness of the sample selection, although this difference is consistent with the fact that more women suffer from knee OA. Additionally, culture, and living environment peculiar to Japanese people are reflected in the text, so caution is required when generalizing the findings across cultures. Second, this is a qualitative study, and its purpose is hypothesis generation. Therefore, although the problems and countermeasures obtained from the interviews could be expected to have some degree of effectiveness, they are issues that need to be verified in the future. Third, the content and intention of the participants' answers were confirmed in the interview, but the cross-validation to confirm if the participants' perceptions were accurately expressed in the manuscript was not performed.

\section{Conclusions}

The perceptions and beliefs of Japanese people with knee OA during conservative care have similarities and differences with those of other countries. Japanese patients with knee OA desired evidence-based information and to connect with other people in the same situation to solve problems related to their condition. Conducting careful interviews and information gathering to clarify and understanding patients' experiences related to knee $O A$ is important. Pain/symptoms experienced by patients with knee $O A$ should be managed by evidence-based information integrating their perceptions and beliefs toward knee OA. Based on the results of this study, we have to develop and evaluate a strategy on how to manage symptoms and disorders experienced by patients with knee OA in future.

\section{Abbreviations}




\section{Declarations}

\section{Ethics approval and consent to participate}

This study was carried out in compliance with the standards laid out in the Declaration of Helsinki, and the study protocol was approved by the research ethics committee of Kio University (H30-36) and Kashiba Asahigaoka Hospital (2018121003). Permission to record the interviews and written informed consent were obtained from all participants before inclusion in the study. A concern when collecting data from patients is that they may feel vulnerable when sharing their experiences with medical staff of the facilities they attend. This potential harm was removed as interviews were conducted by researchers independent of the facilities, and the interview data were transcribed as anonymous data only by the researchers.

\section{Consent to publish}

Not applicable.

\section{Availability of data and materials}

The datasets used and/or analysed during the current study are available from the corresponding author on reasonable request.

\section{Competing interests}

The authors declare that they have no competing interests.

\section{Funding}

This study was not supported by external research funding.

\section{Authors' contributions}

DU, TF, and $\mathrm{KI}$ designed the study. DU, Al, TS, KM, YM, and KI collected, analysed, and interpreted the data. DU and KI wrote and prepared the manuscript. All authors read and approved the final manuscript.

\section{Acknowledgements}

We would like to thank Takanari Kubo PT and Motonari Matsumoto PT for recruiting participants. We would like to thank Editage for English language editing.

\section{References}


1. Chen A, Gupte C, Akhtar K, Smith P, Cobb J: The global economic cost of osteoarthritis: how the UK Compares. Arthritis 2012, 2012:698709.

2. Cross M, Smith E, Hoy D, Nolte S, Ackerman I, Fransen M, Bridgett L, Williams S, Guillemin F, Hill CL et al: The global burden of hip and knee osteoarthritis: estimates from the global burden of disease 2010 study. Ann Rheum Dis 2014, 73(7):1323-1330.

3. Kloppenburg M, Berenbaum F: Osteoarthritis year in review 2019: epidemiology and therapy. Osteoarthritis Cartilage 2020, 28(3):242-248.

4. Muraki S, Oka H, Akune T, Mabuchi A, En-yo Y, Yoshida M, Saika A, Suzuki T, Yoshida H, Ishibashi H et al: Prevalence of radiographic knee osteoarthritis and its association with knee pain in the elderly of Japanese population-based cohorts: the ROAD study. Osteoarthritis Cartilage 2009, 17(9):1137-1143.

5. Bannuru RR, Osani MC, Vaysbrot EE, Arden NK, Bennell K, Bierma-Zeinstra SMA, Kraus VB, Lohmander LS, Abbott JH, Bhandari M et al: OARSI guidelines for the non-surgical management of knee, hip, and polyarticular osteoarthritis. Osteoarthritis Cartilage 2019, 27(11):1578-1589.

6. Bruyère $\mathrm{O}$, Honvo G, Veronese N, Arden NK, Branco J, Curtis EM, Al-Daghri NM, Herrero-Beaumont G, Martel-Pelletier J, Pelletier J-P et al: An updated algorithm recommendation for the management of knee osteoarthritis from the European Society for Clinical and Economic Aspects of Osteoporosis, Osteoarthritis and Musculoskeletal Diseases (ESCEO). Seminars in arthritis and rheumatism 2019, 49(3):337-350.

7. Cuperus N, Smink AJ, Bierma-Zeinstra SM, Dekker J, Schers HJ, de Boer F, van den Ende CH, Vliet Vlieland TP: Patient reported barriers and facilitators to using a self-management booklet for hip and knee osteoarthritis in primary care: results of a qualitative interview study. BMC Fam Pract 2013, 14:181.

8. Lawlor DA, Frankel S, Shaw M, Ebrahim S, Smith GD: Smoking and ill health: does lay epidemiology explain the failure of smoking cessation programs among deprived populations? Am J Public Health 2003, 93(2):266-270.

9. Marshall IJ, Wolfe CD, McKevitt C: Lay perspectives on hypertension and drug adherence: systematic review of qualitative research. BMJ 2012, 345:e3953.

10. MacKay C, Sale J, Badley EM, Jaglal SB, Davis AM: Qualitative study exploring the meaning of knee symptoms to adults ages 35-65 years. Arthritis Care Res (Hoboken) 2016, 68(3):341-347.

11. Richardson JC, Grime JC, Ong BN: 'Keeping going': chronic joint pain in older people who describe their health as good. Ageing Soc 2014, 34(8):1380-1396.

12. Sanders C, Donovan J, Dieppe P: The significance and consequences of having painful and disabled joints in older age: co-existing accounts of normal and disrupted biographies. Sociol Health Illness 2002, 24(2):227-253.

13. Turner AP, Barlow JH, Buszewicz M, Atkinson A, Rait G: Beliefs about the causes of osteoarthritis among primary care patients. Arthritis Rheum 2007, 57(2):267-271.

14. Hobara M: Beliefs about appropriate pain behavior: cross-cultural and sex differences between Japanese and Euro-Americans. Eur J Pain 2005, 9(4):389-393. 
15. Omori M, Dempsey D: Culturally embedded health beliefs, self-care and the use of anti-ageing medicine among Australian and Japanese older adults. Sociol Health Illness 2018, 40(3):523-537.

16. Sathiyamoorthy T, Ali SA, Kloseck M: Cultural factors influencing osteoarthritis care in asian communities: a review of the evidence. J Commun Health 2018, 43(4):816-826.

17. Smith TO, Purdy R, Lister S, Salter C, Fleetcroft R, Conaghan PG: Attitudes of people with osteoarthritis towards their conservative management: a systematic review and meta-ethnography. Rheumatol Int 2014, 34(3):299-313.

18. Wallis JA, Taylor NF, Bunzli S, Shields N: Experience of living with knee osteoarthritis: a systematic review of qualitative studies. BMJ Open 2019, 9(9):e030060.

19. Pope $\mathrm{C}$, Mays N: Reaching the parts other methods cannot reach: an introduction to qualitative methods in health and health services research. BMJ 1995, 311(6996):42-45.

20. O'Brien BC, Harris IB, Beckman TJ, Reed DA, Cook DA: Standards for reporting qualitative research: a synthesis of recommendations. Acad Med 2014, 89(9):1245-1251.

21. Tong A, Sainsbury P, Craig J: Consolidated criteria for reporting qualitative research (COREQ): a 32item checklist for interviews and focus groups. Int J Qual Health Care 2007, 19(6):349-357.

22. Al-Taiar A, Al-Sabah R, Elsalawy E, Shehab D, Al-Mahmoud S: Attitudes to knee osteoarthritis and total knee replacement in Arab women: a qualitative study. BMC Res Notes 2013, 6:406.

23. Brembo EA, Kapstad H, Eide T, Mansson L, Van Dulmen S, Eide H: Patient information and emotional needs across the hip osteoarthritis continuum: a qualitative study. BMC Health Serv Res 2016, 16:88.

24. Hall M, Migay AM, Persad T, Smith J, Yoshida K, Kennedy D, Pagura S: Individuals' experience of living with osteoarthritis of the knee and perceptions of total knee arthroplasty. Physiother Theory Pract 2008, 24(3):167-181.

25. Kao MH, Tsai YF: Illness experiences in middle-aged adults with early-stage knee osteoarthritis: findings from a qualitative study. J Adv Nurs 2014, 70(7):1564-1572.

26. Charmaz K: 'Discovering' chronic illness: using grounded theory. Soc Sci Med 1990, 30(11):11611172.

27. Elliott N, Lazenbatt A: How to recognise a 'quality' grounded theory research study. Aust J Adv Nurs 2005, 22(3):48-52.

28. Swärdh E, Jethliya G, Khatri S, Kindblom K, Opava CH: Approaches to osteoarthritis - A qualitative study among patients in a rural setting in Central Western India. Physiother Theory Pract 2021:1-10.

29. MacKay C, Jaglal SB, Sale J, Badley EM, Davis AM: A qualitative study of the consequences of knee symptoms: 'It's like you're an athlete and you go to a couch potato'. BMJ Open 2014, 4(10):e006006.

30. Vlaeyen JWS, Linton SJ: Fear-avoidance and its consequences in chronic musculoskeletal pain: a state of the art. Pain 2000, 85(3):317-332.

31. Alami S, Boutron I, Desjeux D, Hirschhorn M, Meric G, Rannou F, Poiraudeau S: Patients' and practitioners' views of knee osteoarthritis and its management: a qualitative interview study. PLoS One 2011, 6(5):e19634. 
32. Darlow B, Brown M, Thompson B, Hudson B, Grainger R, McKinlay E, Abbott JH: Living with osteoarthritis is a balancing act: an exploration of patients' beliefs about knee pain. BMC Rheumatol 2018, 2:15.

33. Pouli N, Das Nair R, Lincoln NB, Walsh D: The experience of living with knee osteoarthritis: exploring illness and treatment beliefs through thematic analysis. Disabil Rehabil 2014, 36(7):600-607.

34. Bunzli S, O'Brien P, Ayton D, Dowsey M, Gunn J, Choong P, Manski-Nankervis J-A: Misconceptions and the acceptance of evidence-based nonsurgical interventions for knee osteoarthritis. a qualitative study. Clin Orthop Relat Res 2019, 477(9):1975-1983.

35. Kanavaki AM, Rushton A, Efstathiou N, Alrushud A, Klocke R, Abhishek A, Duda JL: Barriers and facilitators of physical activity in knee and hip osteoarthritis: a systematic review of qualitative evidence. BMJ Open 2017, 7(12):e017042.

36. Gignac MA, Davis AM, Hawker G, Wright JG, Mahomed N, Fortin PR, Badley EM: "What do you expect? You're just getting older": A comparison of perceived osteoarthritis-related and aging-related health experiences in middle- and older-age adults. Arthr Rheum 2006, 55(6):905-912.

37. Kee CC: Living with osteoarthritis: insiders' views. Appl Nurs Res 1998, 11(1):19-26.

38. McHugh GA, Silman AJ, Luker KA: Quality of care for people with osteoarthritis: a qualitative study. J Clin Nurs 2007, 16(7B):168-176.

39. Parsons GE, Godfrey H, Jester RF: Living with severe osteoarthritis while awaiting hip and knee joint replacement surgery. Musculoskeletal Care 2009, 7(2):121-135.

40. Bennell KL, Dobson F, Hinman RS: Exercise in osteoarthritis: moving from prescription to adherence. Best Pract Res Clin Rheumatol 2014, 28(1):93-117.

41. Tudor-Locke C, Craig CL, Aoyagi Y, Bell RC, Croteau KA, De Bourdeaudhuij I, Ewald B, Gardner AW, Hatano Y, Lutes LD et al: How many steps/day are enough? For older adults and special populations. Int J Behav Nutr Phys Act 2011, 8:80.

42. Bower KN, Frail D, Twohig PL, Putnam W: What influences seniors' choice of medications for osteoarthritis? Qualitative inquiry. Can Fam Physician 2006, 52:342-343.

43. Chan KKW, Chan LWY: A qualitative study on patients with knee osteoarthritis to evaluate the influence of different pain patterns on patients' quality of life and to find out patients' interpretation and coping strategies for the disease. Rheumatology Reports 2011, 3(1):e3.

44. Mann C, Gooberman-Hill R: Health care provision for osteoarthritis: concordance between what patients would like and what health professionals think they should have. Arthritis Care Res (Hoboken) 2011, 63(7):963-972.

45. Keysor JJ, Sparling JW, Riegger-Krugh C: The experience of knee arthritis in athletic young and middle-aged adults: an heuristic study. Arthritis Care Res 1998, 11(4):261-270.

46. Harada K, Masumoto K, Kondo N: Exercising alone or exercising with others and mental health among middle-aged and older adults: Iongitudinal analysis of cross-lagged and simultaneous effects. J Phys Act Health 2019, 16(7):556-564. 
47. Kanamori S, Takamiya T, Inoue S, Kai Y, Kawachi I, Kondo K: Exercising alone versus with others and associations with subjective health status in older Japanese: The JAGES Cohort Study. Sci Rep 2016, 6:39151.

\section{Tables}

Table 1. Interview guide 


\section{Introduction}

Please tell me if you remember the first time you noticed the symptom (pain).

\section{Perceptions}

How did you feel when you were diagnosed with knee osteoarthritis?

What are your thoughts on your current symptoms (pain)?

Do you think this symptom (pain) will remain in the future? Why do you think so?

Physical

What was the most important (or first) physical concern when you were diagnosed with knee osteoarthritis?

How is your knee condition now?

Do you want to reduce pain further with surgery?

Have you ever been happy with the pain?

Is there any difference between pain in other areas and knee pain?

\section{Life}

What was the most important (first) concern in your life when you were diagnosed with knee osteoarthritis?

How has knee osteoarthritis affected your daily life?

Are you doing anything to mitigate those effects?

\section{Information}

What do or did you know about knee osteoarthritis?

Where and how did you collect information on how to deal with illness and pain?

What kind of information has been useful so far?

What kind of information did you want at the time of your first visit (or onset)?

What kind of information do you want now? 


\section{Others}

What kind of services do you currently receive (hospitals, outpatients)?

What kind of service are you looking for?

With whom do you talk about your current symptoms (pain, etc.)?

How do you explain your symptoms to others?

Do you keep a record of your illness? Why?

\section{Summary}

If you could go back in time and do something differently to prevent or manage knee OA, what would it be?

Do you have any other experience or feelings on this subject that you would like to talk about?

Table 2. Demographic data for the nine participants involved in the study 


\begin{tabular}{|c|c|c|c|c|c|c|c|}
\hline ID & Sex & $\begin{array}{l}\text { Age } \\
\text { (years) }\end{array}$ & $\begin{array}{l}\text { Symptom } \\
\text { duration }\end{array}$ & $\begin{array}{l}\text { Place } \\
\text { recruited }\end{array}$ & Employment status & $\begin{array}{l}\text { KL grade } \\
(\mathrm{R} / \mathrm{L})\end{array}$ & $\begin{array}{l}\text { Pain } \\
(\mathrm{R} / \mathrm{L})\end{array}$ \\
\hline A & $\mathrm{W}$ & 70 & $3 \mathrm{mos}$ & $\begin{array}{l}\text { Hospital } \\
\text { in suburban } \\
\text { area }\end{array}$ & $\begin{array}{l}\text { Housewife } \\
\text { (real estate business, } \\
\text { retired) }\end{array}$ & $4 / 0$ & $5 / 0$ \\
\hline B & M & 67 & 5 yrs & $\begin{array}{l}\text { Hospital } \\
\text { in suburban } \\
\text { area }\end{array}$ & Carpenter & TKA/TKA & $1 / 1$ \\
\hline $\mathrm{C}$ & M & 77 & $10 \mathrm{yrs}$ & $\begin{array}{l}\text { Hospital } \\
\text { in suburban } \\
\text { area }\end{array}$ & $\begin{array}{l}\text { Volunteer work } \\
\text { (office worker, retired) }\end{array}$ & $3 / 3$ & $4 / 4$ \\
\hline $\mathrm{D}$ & W & 75 & $10 \mathrm{yrs}$ & $\begin{array}{l}\text { Hospital } \\
\text { in suburban } \\
\text { area }\end{array}$ & Volunteer work, housewife & $3 / 3$ & $3 / 4$ \\
\hline $\mathrm{E}$ & $\mathrm{W}$ & 76 & $\begin{array}{l}\text { Over } 25 \\
\text { yrs }\end{array}$ & $\begin{array}{l}\text { Hospital } \\
\text { in suburban } \\
\text { area }\end{array}$ & $\begin{array}{l}\text { Housewife } \\
\text { (Family operated business, } \\
\text { retired) }\end{array}$ & TKA $/ 4$ & $0 / 5$ \\
\hline $\mathrm{F}$ & $\mathrm{W}$ & 83 & $3 \mathrm{mos}$ & $\begin{array}{l}\text { Clinic } \\
\text { in urban area }\end{array}$ & Housewife & $2 / 2$ & $5 / 5$ \\
\hline G & W & 74 & $3 \mathrm{mos}$ & $\begin{array}{l}\text { Clinic } \\
\text { in urban area }\end{array}$ & Housewife & $1 / 1$ & $2 / 2$ \\
\hline $\mathrm{H}$ & W & 67 & 25 yrs & $\begin{array}{l}\text { Hospital } \\
\text { in suburban } \\
\text { area }\end{array}$ & Part-time university lecturer & TKA/TKA & $1 / 1$ \\
\hline I & W & 80 & $1 \mathrm{yr}$ & $\begin{array}{l}\text { Health care } \\
\text { program } \\
\text { in suburban } \\
\text { area }\end{array}$ & $\begin{array}{l}\text { Housewife } \\
\text { (medical professor, tai-chi } \\
\text { instructor, retired) }\end{array}$ & $0 / 2$ & $0 / 3$ \\
\hline
\end{tabular}

\#Pain is described by Numerical Rating Scale. ${ }^{\$}$ KOOS is described as total score.

KL grade; Kellgren-Lawrence grade, KOOS; Knee injury and Osteoarthritis Outcome Score, L; Left, M; Man, NRS; Numerical Rating Scale, R; Right, TKA; Total Knee Arthroplasty, W; Woman

\section{Figures}




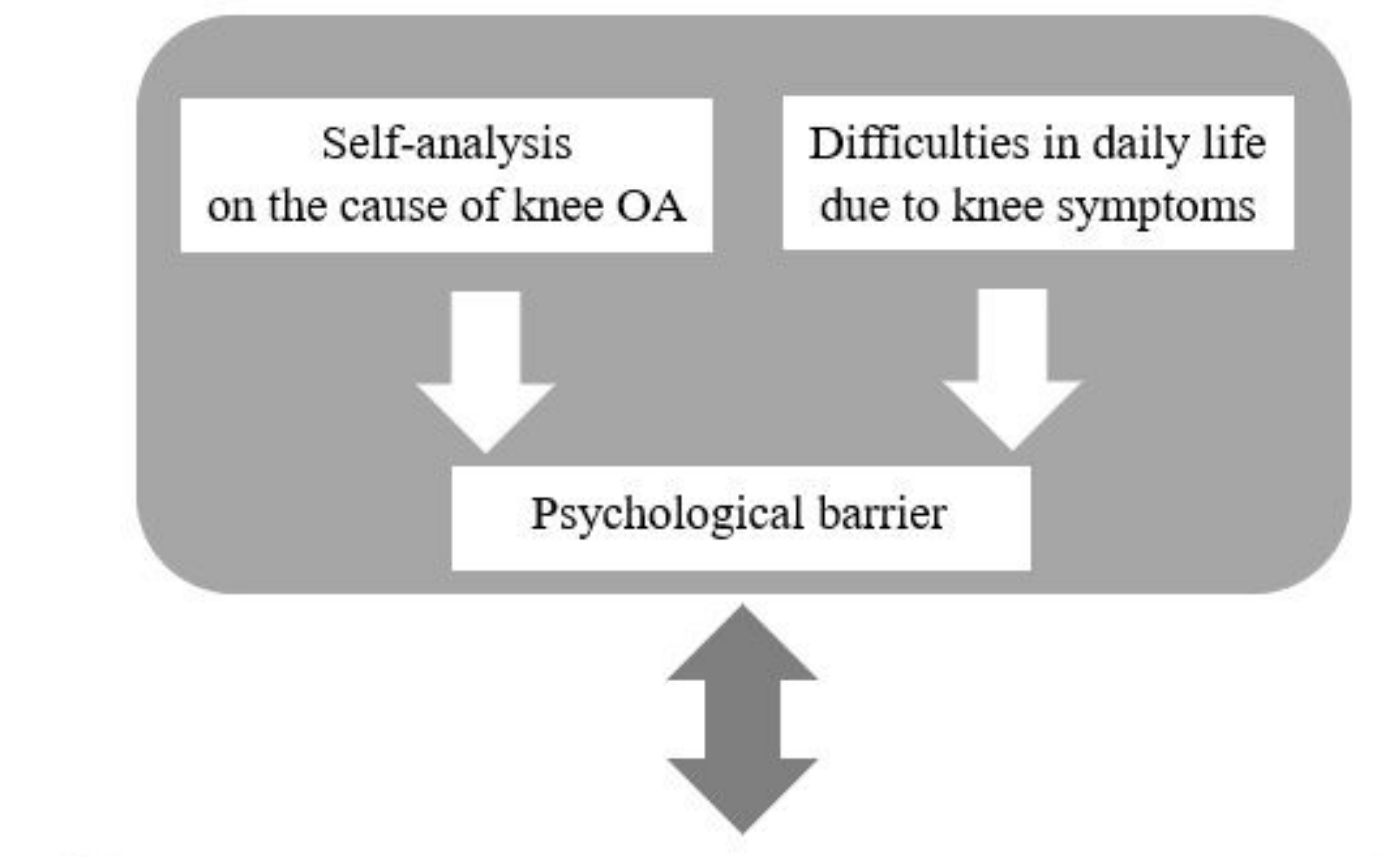

How to deal with knee pain and difficulty in moving

Information considered useful to cope with knee OA

Importance of connecting with others

Figure 1

Relationship between categories

\section{Supplementary Files}

This is a list of supplementary files associated with this preprint. Click to download.

- Additionalfile.docx 